\title{
The Use of Technology to Enhance Health
}

\author{
Wendy Nilsen, PhD \\ Office of Behavioral and Social Sciences Research, National Institutes of Health, Bethesda, MD, USA.
}

J Gen Intern Med 30(8):1047-8

DOI: $10.1007 / \mathrm{s} 11606-015-3307-5$

(c) Society of General Internal Medicine 2015

$\mathrm{T}$ he article by Donna Zulman and colleagues in this issue of the Journal of General Internal Medicine ${ }^{1}$ provides important information in support of our understanding of the utility and value of eHealth - more broadly known as health information technology — for people with multiple chronic conditions (MCC).

More than one in four Americans lives with the burden of two or more ongoing health conditions. ${ }^{2}$ As the population ages, the number of people living with MCC is growing dramatically, ${ }^{3}$ and their medical costs account for $75 \%$ of health care spending in the United States. Among the Medicare older adult population, over $90 \%$ of health care spending is devoted to people with MCC. ${ }^{4}$ However, research shows that this heavy expenditure has not resulted in the desired improvements in the quality of life of the people with MCC. $^{4}$

In this environment, eHealth-including computers, Internet, mobile technologies and sensors - may have some potential to improve health and enhance the quality of life, while also reducing costs. eHealth technology allows for smart distillation and distribution of information in a personalized format that should support patients and providers alike, especially with the complex issues of managing multiple chronic conditions.

Zulman and colleagues' work explores how a group of 53 people with MCC at either a Veterans Affairs or an academic medical center used technology. Their survey and focus groups studied the technology usage and needs of people with MCC. Not surprisingly, the group found that participants' eHealth usage and requirements targeted three specific areas: managing, coordinating, and generating information to support their health. Management of information focused on collecting and organizing schedules, exploring treatment effects and medication interactions, and collating these data for the patient, caregiver and health care team. Coordinating information stressed the need for communication between the many systems and providers involved with people with MCC. Finally, generating information described people's collection of health information from disparate sources so that they could

Published online April 8, 2015 develop specialized knowledge about their diseases and treatments, both for their own needs and to share with providers.

The focus of Zulman and colleagues' article is especially important given multiple recent articles in the popular press noting that eHealth and mobile health have not been fulfilling their potential. The overriding message is that health information technology, regardless of format, has to move away from being just exciting technology for a select group of tech-savvy individuals and become a product that fits the needs of the population and merges an understanding of clinical science and consumer behavior to create useful tools. This emerging literature also emphasizes the need to conceptualize and design health information technology around the needs of actual end-users. Instead of developing tools that are based on the idea of passive patients waiting for health technology to affect their health and care, we should view the end-users as consumers that are deciding how to best use their time and resources to improve their lives. In this model, we are forced to think of health in the broader context of someone's life, including how they think about and manage their health.

Under this paradigm, usefulness is key, because those who are most in need of support in managing their health, such as people with MCC, likely do not have the time or energy to tolerate poorly designed technology. For example, a blood pressure monitor that repeatedly un-syncs from a phone or cannot upload data in a format that is useful for a provider will only frustrate and discourage people from using eHealth technology.

For many potential users, especially those managing the demands of MCC, a device that assumes you have nothing more to do than think about your technology, or that serially prompts you to take each of your eight medications, may have little or no chance in the marketplace. This also assumes that users have an understanding of and are comfortable with technology, which may not be true for patients who are older or in poorer health. As Patel, Ash and Volpp ${ }^{5}$ recently noted, technology is no more than a tool in our quest to enhance health and improve quality of life. The technology itself is not magic, and for it to be successful, we will need expertise from many disciplines, both within and outside the health professions. .

Moving eHealth forward will require a firm understanding of behavior, including the mediators and moderators of everyday activities and decisions that surround health. With this broad knowledge, we can bring in experts specializing in user 
experience, human-computer interaction, human factors, and other related disciplines who will partner with health and technology experts to create eHealth tools that improve health outcomes for persons with a range of diseases, including MCC. ${ }^{6}$ Based on our best science, these tools will fit into people's lives and will be useful. For people with MCC, this means not only usability in the conventional sense of technology design, but also usefulness at managing multiple diseases more effectively and efficiently than with individual tools.

Much of what patients report wanting, however, does not rely on the "smartness" of a smartphone. As Zulman and colleagues note, the participants in their study were looking for technology to solve some of the most basic problems, such as facilitating communication among providers, making it easier to explore and combine information for multiple conditions in one search, and drawing information in a standardized format of electronic health record. Working across disciplines and with patients will allow us to develop a range of technologies and to build these tools.

Work like that of Zulman and colleagues ${ }^{1}$ leads the way to a future where technology and health will merge to provide services that better support and enhance the health of populations, including those with MCC. Future research should expand on their work, to study the needs of those who only marginally use or have not adopted technology. By developing a better understanding of the potential users of technology and their needs, we can develop eHealth tools that support our ultimate goal: enhancing health and improving quality of life.

Disclaimer: The conclusions in this report are those of the author and do not necessarily reflect the position of the National Institutes of Health.

Author: Wendy Nilsen, PhD; Office of Behavioral and Social Sciences Research, National Institutes of Health, Bethesda, MD, USA (e-mail: nilsenwj@od.nih.gov).

\section{REFERENCES}

1. Zulman DM, Jenchura EC, Cohen DM, Lewis ET, Houston TK, Asch SM. How can eHealth technology address challenges related to multimorbidity? Perspectives from patients with multiple chronic conditions. J Gen Intern Med. 2015. doi:10.1007/s11606-015-3222-9.

2. Ward BW, Schiller JS. Prevalence of multiple chronic conditions among US adults: estimates from the National Health Interview Survey, 2010. Prev Chronic Dis. 2013; 10:E65.

3. Institute of Medicine. Living well with chronic illness: a call for public health action. Washington: National Academy Press; 2012.

4. Machlin SR, Soni A. Health care expenditures for adults with multiple treated chronic conditions: estimates from the Medical Expenditure Panel Survey, 2009. Prev Chronic Dis. 2013;10:E63.

5. Patel MS, Asch DA, Volpp KG. Wearable devices as facilitators, not drivers, of health behavior change. JAMA. 2015;313(5):459-60.

6. Johnson CM, Johnson TR, Zhang J. A user-centered framework for redesigning health care interfaces. J Biomed Inform. 2005;38(1):75-87. 